PROCEEDINGS OF THE

AMERICAN MATHEMATICAL SOCIETY

Volume 138, Number 1, January 2010, Pages 101-104

S 0002-9939(09)10052-7

Article electronically published on August 24, 2009

\title{
A SUM-DIVISION ESTIMATE OF REALS
}

\author{
LIANGPAN LI AND JIAN SHEN \\ (Communicated by Ken Ono)
}

\begin{abstract}
Let $A$ be a finite set of positive real numbers. We present a sumdivision estimate:

$$
|A+A|^{2}|A / A| \geq \frac{|A|^{4}}{4} .
$$
\end{abstract}

\section{INTRODUCTION}

Let $A$ be a finite set of positive real numbers throughout. The sum-set, productset and ratio-set of $A$ are defined respectively to be

$$
\begin{aligned}
A+A & =\{a+b: a, b \in A\}, \\
A A & =\{a b: a, b \in A\}, \\
A / A & =\{a / b: a, b \in A\} .
\end{aligned}
$$

A famous conjecture of Erdös and Szemerédi [6] asserts that for any $\alpha<2$, there exists a constant $C_{\alpha}>0$ such that

$$
\max \{|A+A|,|A A|\} \geq C_{\alpha}|A|^{\alpha} .
$$

In a series of papers $[1,2,1,11,12,13$, upper bounds on $\alpha$ were found by many authors. One highlight in this direction was a proof by Elekes 2, that $\alpha$ can be taken to be $\frac{5}{4}$. His argument utilized a clever application of the Szemerédi-Trotter theorem on point-line incidences. Recently, using the concept of multiplicative energy and an ingenious geometric observation, Solymosi [14] obtained that if $A$ is not a singleton, then

$$
|A+A|^{2}|A A| \geq \frac{|A|^{4}}{4\left\lceil\log _{2}|A|\right\rceil}
$$

which yields

$$
\max \{|A+A|,|A A|\} \geq \frac{|A|^{4 / 3}}{2\left\lceil\log _{2}|A|\right\rceil^{1 / 3}} .
$$

One cannot completely drop the logarithmic term in (2), since if we choose $\widetilde{A}=$ $\{1,2, \ldots, n\}$, then [4, 5, 8, 15 ]

$$
|\widetilde{A} \widetilde{A}|=\frac{n^{2}}{(\ln n)^{\beta+o(1)}}, \quad \beta=1-\frac{1+\ln \ln 2}{\ln 2}=0.0860713 \ldots .
$$

Received by the editors May 12, 2009, and, in revised form, May 18, 2009.

2000 Mathematics Subject Classification. Primary 11B75.

Key words and phrases. Sum-product estimate, sum-division estimate.

(C)2009 American Mathematical Society Reverts to public domain 28 years from publication 
There is a subtle difference between $|\widetilde{A} \widetilde{A}|$ and $|\widetilde{A} / \widetilde{A}|$. In fact, Elekes and Ruzsa [3] showed that there exists a universal constant $\gamma>0$ such that

$$
|A+A|^{6}|A / A| \geq \gamma|A|^{8}
$$

which yields

$$
|\widetilde{A} / \widetilde{A}| \geq \frac{\gamma}{64}|\widetilde{A}|^{2}
$$

by choosing $A=\widetilde{A}$. This leads to a natural question: how to give a joint estimate on $|A+A|$ and $|A / A|$ ? It is not difficult to use the Szemerédi-Trotter theorem on point-line incidences to show that

$$
|A+A \| A / A| \geq C|A|^{5 / 2}
$$

holds for some universal constant $C>0$. Besides, if we carefully analyze Solymosi's proof of (1), then

$$
|A+A|^{2}|A / A| \geq \frac{|A|^{4}}{4\left\lceil\log _{2}|A|\right\rceil} .
$$

The main purpose of this paper is to drop the term $\left\lceil\log _{2}|A|\right\rceil$ in (6) .

Theorem 1. Let $A$ be a finite set of positive real numbers. Then

$$
|A+A|^{2}|A / A| \geq \frac{|A|^{4}}{4} .
$$

This implies a sum-division estimate

$$
\max \{|A+A|,|A / A|\} \geq \frac{|A|^{4 / 3}}{2} .
$$

There is an explanation of Theorem 1 in plane geometry. View $\mathbb{R}^{2}$ naturally as the complex plane $\mathbb{C}$. Given a finite set $A$ of positive real numbers, denote by $\operatorname{Rad}(A \times A)$ and $\operatorname{Ang}(A \times A)$ respectively the radius-set and the angle-set of $A \times A$. Applying Theorem 1 with $\widehat{A}=\left\{a^{2}: a \in A\right\}$ yields

$$
\max \{|\operatorname{Rad}(A \times A)|,|\operatorname{Ang}(A \times A)|\} \geq \frac{|A|^{4 / 3}}{2} .
$$

This shows the angle-set and the radius-set of $A \times A$ cannot be small simultaneously.

\section{Proof of the main Result}

Suppose $|A / A|=y$ and $A / A=\left\{z_{i}\right\}_{i=1}^{y}$. Suppose $z_{i}$ has $m_{i}$ representations in $A \times A$, that is,

$$
m_{i}=\left|\left\{(a, b) \in A \times A: \frac{a}{b}=z_{i}\right\}\right| \quad(i=1,2, \ldots, y) .
$$

Without loss of generality we may order all $m_{i}$ 's as follows:

$$
m_{1} \leq m_{2} \leq \cdots \leq m_{y}
$$

Since $|A|^{2}=\sum_{i=1}^{y} m_{i}$, there exists a unique integer $k, 1 \leq k \leq y$, such that

$$
\sum_{i=1}^{k-1} m_{i}<\frac{|A|^{2}}{2} \leq \sum_{i=1}^{k} m_{i} \leq k m_{k} .
$$

Hence

$$
|A / A| \geq k \geq \frac{|A|^{2}}{2 m_{k}}
$$


and

$$
\sum_{i=k}^{y} m_{i}=\left(|A|^{2}-\sum_{i=1}^{k-1} m_{i}\right) \geq \frac{|A|^{2}}{2} .
$$

By (7) and Solymosi's geometric observation [14,

$$
|A+A|^{2}=|(A \times A)+(A \times A)| \geq m_{k} \sum_{i=k}^{y} m_{i} .
$$

Multiplying (8), (9) and (10) yields

$$
|A+A|^{2}|A / A| \geq \frac{|A|^{4}}{4} .
$$

This proves Theorem 1 .

Remark. Let $F_{n}=\{a / q: 1 \leq a \leq q \leq n,(a, q)=1\}$ be the set of Farey fractions of order $n$. It is well-known ([10]) that $\left|F_{n}\right| \sim \frac{3}{\pi^{2}} n^{2}$ as $n \rightarrow \infty$. Besides, it is not difficult to deduce from (3) (see also [8, 9]) that

$$
\max \left\{\left|F_{n}+F_{n}\right|,\left|F_{n}-F_{n}\right|,\left|F_{n} F_{n}\right|,\left|F_{n} / F_{n}\right|\right\} \leq \frac{n^{4}}{(\ln n)^{\beta+o(1)}} \quad(n \rightarrow \infty) .
$$

This shows generally that one cannot expect the estimate

$$
\max \{|A+A|,|A / A|\} \asymp|A|^{2} \quad(|A| \rightarrow \infty) .
$$

We thank Dimitris Koukoulopoulos for communicating this example to us.

\section{ACKNOWLEDGMENTS}

The first author's research was supported by the Mathematical Tianyuan Foundation of China (No. 10826088). The second author's research was partially supported by NSF (CNS 0835834) and Texas Higher Education Coordinating Board (ARP 003615-0039-2007).

\section{REFERENCES}

[1] Y.-G. Chen, On sums and products of integers, Proc. Amer. Math. Soc. 127 (1999), 19271933. MR.1600124 (99j:11022)

[2] Gy. Elekes, On the number of sums and products, Acta Arith. 81 (1997), 365-367. MR:1472816 (98h:11026)

[3] Gy. Elekes and I. Z. Ruzsa, Few sums, many products, Studia Sci. Math. Hungar. 40 (2003), 301-308. MR2036961 (2005d:11011)

[4] P. Erdős, Some remarks on number theory, Riveon Lematematika 9 (1955), 45-48. MR0073619 (17,460d)

[5] P. Erdős, An asymptotic inequality in the theory of numbers, Vestnik Leningrad. Univ. 15 (1960), 41-49. MR0126424 (23:A3720)

[6] P. Erdős and E. Szemerédi, On sums and products of integers, Studies in Pure Mathematics, 213-218, Birkhäuser, Basel, 1983. MR0820223 (86m:11011)

[7] K. Ford, Sums and products from a finite set of real numbers, Ramanujan J. 2 (1998), 59-66. MR.1642873 (99i:11014)

[8] K. Ford, The distribution of integers with a divisor in a given interval, Ann. of Math. (2) 168 (2008), 367-433. MR2434882

[9] A. K. Haynes and K. Homma, The group ring of $\mathbb{Q} / \mathbb{Z}$ and an application of a divisor problem, Proc. Amer. Math. Soc. 137 (2009), 1285-1293. MR2465650

[10] L. K. Hua, Introduction to Number Theory, translated from the Chinese by Peter Shiu. Springer-Verlag, Berlin-New York, 1982. MR0665428 (83f:10001) 
[11] M. B. Nathanson, On sums and products of integers, Proc. Amer. Math. Soc. 125 (1997), 9-16. MR1343715 (97c:11010)

[12] J. Solymosi, On sum-sets and product-sets of complex numbers, J. Thèor. Nombres Bordeaux 17 (2005), 921-924. MR2212132 (2006m:11011)

[13] J. Solymosi, On the number of sums and products, Bull. London Math. Soc. 37 (2005), 491-494. MR2143727 (2006c:11021)

[14] J. Solymosi, Bounding multiplicative energy by the sumset, Adv. Math. (2009), doi:10.1016/ j.aim.2009.04.006

[15] G. Tenenbaum, Sur la probabilité qu'un entier possède un diviseur dans un intervalle donné, Compositio Math. 51 (1984), 243-263. MR0739737 (86c:11009)

Department of Mathematics, Shanghai Jiao Tong University, Shanghai 200240, PeoPle's Republic of China - and - Department of Mathematics, Texas State University, San Marcos, Texas 78666

E-mail address: liliangpan@yahoo.com.cn

Department of Mathematics, Texas State University, San Marcos, Texas 78666

E-mail address: js48@txstate.edu 